\title{
Transatlantica
}

Revue d'études américaines. American Studies Journal

\section{Centenaire de Eudora Welty à Jackson}

16-19 avril 2009

\section{Isabelle Mattéi}

\section{(2) OpenEdition}

Journals

Édition électronique

URL : https://journals.openedition.org/transatlantica/4667

DOI : $10.4000 /$ transatlantica.4667

ISSN : $1765-2766$

Éditeur

Association française d'Etudes Américaines (AFEA)

\section{Référence électronique}

Isabelle Mattéi, "Centenaire de Eudora Welty à Jackson », Transatlantica [En ligne], 2 | 2009, mis en

ligne le 02 février 2010, consulté le 31 janvier 2023. URL : http://journals.openedition.org/

transatlantica/4667; DOI : https://doi.org/10.4000/transatlantica.4667

Ce document a été généré automatiquement le 31 janvier 2023.

\section{(c) $(†)$}

Creative Commons - Attribution - Pas d'Utilisation Commerciale - Pas de Modification 4.0 International - CC BY-NC-ND 4.0

https://creativecommons.org/licenses/by-nc-nd/4.0/ 


\section{Centenaire de Eudora Welty à Jackson}

16-19 avril 2009

Isabelle Mattéi

1 En avril dernier, la ville natale de Eudora Welty, Jackson, dans le Mississippi, a rendu hommage à sa plus célèbre représentante, en organisant à l'occasion du centième anniversaire de sa naissance, un colloque international qui a réuni, sous l'égide d'Harriet Pollack (Bucknell University) les plus éminents spécialistes de cette auteure du Sud, romancière, nouvelliste, mais aussi photographe de talent. Ces rencontres à Jackson se sont déroulées en même temps que le Southern Literary Festival, et ont donné lieu, par ailleurs, à de nombreuses manifestations culturelles et sociales.

2 Ainsi la première matinée a été consacrée à la visite de la maison de Eudora Welty sur Pinehurst Street, aujourd'hui transformée en musée, où elle passa la majeure partie de sa vie. Accompagnés par Mary Alice White, sa nièce, les participants du colloque ont pu s'imprégner de l'atmosphère de la maison familiale, découvrir la bibliothèque avec ses disques de jazz et sa collection de livres, comme les Dickens qu'elle avait reçus en cadeau de sa mère et qu'elle affectionnait tout particulièrement. Puis au premier étage, sa chambre et son bureau devant la fenêtre, d'où elle pouvait observer la rue et les passants, y puisant sans doute une partie de son inspiration. Enfin la visite du jardin où elle aimait se promener au milieu des fleurs fut une agréable conclusion à cette matinée du souvenir.

\section{Communications}

3 Le colloque, qui ne portait pas sur un thème unique, s'est déroulé sur quatre jours articulés autour de dix sessions distinctes. 


\section{6 avril}

4 Après l'accueil chaleureux des participants par Harriet Pollack et l'intervention inaugurale de Peggy Prenshaw, doyenne de Millsaps College, sur l'écriture de Welty, la première session, intitulée "From the Archives", a permis d'entendre trois interventions: Michael Kreyling (Vanderbilt University), critique weltien qui a insisté sur les données biographiques et mis en lumière les rapports particuliers qu'entretenaient Eudora Welty et son agent Diarmund Russell dans son livre Author and Agent (1991), a expliqué en prenant du recul sur son propre ouvrage, comment le désir et la crainte de ce désir sauvage, qui reste inassouvi, constituait le véritable sujet des nouvelles, initialement identifié à la « Natchez Trace ».

5 Julia Eichelberg (Charleston College) s'est intéressée quant à elle, aux nombreuses lettres de Welty sur le jardinage, qui témoignent de sa passion pour les fleurs mais qui, au-delà de l'aspect divertissant, prennent une signification plus profonde : le jardinage est lié à d'autres formes de créativité, et peut même, comme dans la nouvelle éponyme du recueil A Curtain of Green, revêtir un pouvoir de transgression. On peut citer à cet égard le dernier livre de Patti Carr Black : Eudora Welty's World: Words on Nature (2005) où elle associe avec bonheur les aquarelles du Mississipi peintes par Robin Whitfield et les mots empruntés à l'œuvre de Eudora Welty.

6 Ann Romines (George Washington University) enfin, a évoqué le rôle de l'amitié dans la vie et les textes de Welty. Ces "réseaux » d'amitié, particulièrement évidents dans le second recueil de nouvelles, The Wide Net, sont essentiellement masculins, les femmes étant plus souvent solitaires que solidaires. Les nombreuses lettres et visites que Welty recevait à Jackson prouvent à quel point l'amitié était importante à ses yeux. Pour s'en persuader, il faut lire la biographie de Suzanne Marrs, Eudora Welty : A Biography.

7 La session suivante, "Medusa, Circe and Cixous", a été consacrée aux figures mythologiques qui parsèment son œuvre, en particulier The Golden Apples. John Lowe (Louisiana State University) a souligné le pouvoir subversif du comique chez Welty, comique qualifié de «cosmique " par les nombreuses références bibliques et le motif central de la Méduse. Elizabeth Crews, doctorante à Georgia State University, a étudié de son côté la nouvelle "A Curtain of Green » dans une perspective féministe, en appliquant le concept d' « écriture féminine » de Cixous, à l'héroïne de la nouvelle, Mrs Larkin. Patricia Bradley (Middle Tennessee State University) enfin, a conclu cette première journée en s'intéressant aux figures de Perséphone et de Circé, dans l'œuvre de Welty.

\section{7 avril}

8 La première session de la matinée "Welty and other Southern Writers" a exploré la dimension intertextuelle de l'oeuvre de Welty, et confronté son écriture avec celle d'autres écrivains du Sud, tout en évoquant certains de ses textes moins commentés, comme « Where is the Voice coming from?».

9 Anita Garner (University of North Alabama) et Sharon Baris (Université de Bar-Ilan, Israel) ont toutes les deux rappelé le contexte de ce récit assez singulier dans le corpus de Welty, écrit en une nuit après le meurtre de Medgar Evers, et pour lequel elle avait dû entrer dans la peau et l'esprit d'un meurtrier qui lui était particulièrement 
répugnant (« into the mind and inside the skin of a character who could hardly have been more alien or repugnant to me. » One Writer's Beginnings).

Pour Anita Garner, la voix narrative (le meurtrier) est au centre même de la nouvelle ; c'est la raison pour laquelle elle compare son écriture à celle de Truman Capote et au courant du "nouveau journalisme ", tout en montrant que Welty va bien au delà en le transformant en mythe. Sharon Baris quant à elle, s'est intéressée en outre à un autre texte moins connu de Welty : «The Shoebird», un récit pour enfants publié en 1964.

11 Mae Claxton Miller (Western Carolina University) a ensuite rapproché Eudora Welty et Barbara Kingsolver à travers leurs récits centrés sur la nature, les femmes et la perte. La nature chez ces deux auteures n'est pas seulement l'environnement de l'histoire, mais une force vitale liée à la communauté, l'humain, en même temps qu'à l'inhumain.

Enfin, mémoire et passé ont été explorés sous un angle nouveau par Susan Donaldson (College of William and Mary) qui a renouvelé les perspectives en montrant comment Welty rompait avec la tradition nostalgique du Sud. Les personnages de Katherine Ann Porter, Carson McCullers, et Margaret Mitchell sont toujours empêtrés dans les souvenirs du passé, alors que dans " The Burning », l'héroïne de Eudora Welty, elle, est davantage tournée vers l'avenir, ce texte pouvant, selon Donaldson, être considéré comme une parodie de Gone with the Wind.

session suivante "Welty and Aesthetics " a donné lieu à trois communications : Pearl McHaney (Georgia State University) a démontré qu'une esthétique surréaliste était à l'œuvre dans les nouvelles "The Winds » et "A Sketching Trip», et que ce qu'on a coutume de nommer "grotesque » pouvait être lu comme «surréel». La nouvelle, qui repose en effet sur la toute puissance du rêve de Josie, montre, selon elle, le mécanisme des pensées, et non des significations.

14 Pour Paul McCann (Del Mar College), c'est une esthétique du jazz qui définit la nouvelle «Powerhouse ». En effet, ce texte est en rupture stylistique avec les autres textes de Welty, et McCann l'apparente à l'écriture de Jack Kerouac, également fondée sur une forme stylistique spontanée. "Powerhouse », selon McCann, fait le pont entre les récits jazz des années trente, et les textes de Kerouac. Il salue finalement l'audace de Welty qui a réussi à transposer le jazz en écriture, rédigeant sa nouvelle un peu comme une improvisation en jazz.

15 Dans l'une de ses lettres, Welty avait dit du court roman The Robber Bridegroom qu'il s'était pratiquement écrit tout seul. Barbara Sylvester (Western Washington University) s'est intéressée à l'adaptation qu'en a faite le dramaturge et scénariste Alfred Uhry, généralement fidèle, selon elle, au texte original malgré quelques exagérations, notamment en ce qui concerne l'humour.

16 Ce dernier, en fin de matinée, évoqua son travail d'écriture théâtrale et cinématographique, ainsi que le film Driving Miss Daisy pour lequel il eut l'Oscar du meilleur scénario en 1990.

17 Conjointement avec le Southern Literary Festival, l'après-midi a débuté par une série de témoignages personnels qui ont corrigé l'image de femme casanière, austère et solitaire de Welty. Tour à tour Suzanne Marrs, Patti Carr Black et Mary Alice White ont évoqué la grande dame du Sud avec affection, rappelant qu'elle avait en fait beaucoup voyagé, soulignant aussi son humour ainsi que sa passion pour le langage et les jeux de mots. William Winter, ancien gouverneur du Mississippi, a pour sa part évoqué la période où Miss Welty enseignait à Millsaps, et Richard Ford s'est attaché à expliquer la façon dont 
une histoire prend vie et forme, est in-carnée, montrant comment le paysage s'incarne, prend chair dans les récits de Eudora Welty.

Ensuite, la romancière Elizabeth Spencer a ravi l'auditoire en lisant avec son inimitable accent du Sud un extrait de Losing Battles puis d'une de ses propres nouvelles («First Dark »). Ann Patchett a conclu la journée par la lecture d'un passage du roman qu'elle était en train d'écrire.

\section{8 avril}

19 La première session du samedi «Bridging differences » a abordé la question de l'identité et de l'altérité, à travers trois interventions autour de la voix, de la table, et du masque.

20 James Andrew Crank (Northwestern State University) a montré comment, dans The Ponder Heart, se livre une véritable guerre des voix, la voix du personnage principal Daniel Ponder étant dominée tout au long du récit par celle de la narratrice Edna Earle jusqu'au dénouement où seule la voix de Ponder peut, par son pouvoir d'empathie, réconcilier les différences de classe et donner in fine une cohérence à l'histoire.

21 Abigael Lundilius (University of South Carolina) a ensuite analysé la nouvelle "Moon Lake» dans une perspective psychanalytique en expliquant comment l'identité féminine est définie par rapport à la communauté : le moi individuel s'efface devant le moi communautaire, et la table est un lieu investi d'une dimension à la fois charnelle (nourriture), et symbolique et religieuse (lieu de sacrifice qui devient sacrement).

22 Anne Rashid (Carlow University) enfin, s'est intéressée aux relations complexes entre l'auditoire noir et blanc et Powerhouse dans la nouvelle éponyme. Eudora Welty y montre la vie de l'artiste dérangeant aux multiples masques qui résiste aux interprétations réductrices du public, et manipule aussi bien son audience que le lecteur du texte, les tensions musicales et raciales étant transfigurées par le pouvoir rédempteur de l'art.

23 Dans la session suivante, « Maps, rivers and drums », Joseph Millchap (Western Kentucky University) a montré comment la nature était inscrite au cœur de l'œuvre de Welty (même dans le nom des personnages), et a mis en relief le rôle essentiel des fleuves jusque dans The Golden Apples. Adrienne Akins s'est appuyée sur les théories postcoloniales pour commenter le roman Losing Battles, récit cartographique où se croisent les lieux et la mémoire. Et Corrie Catlett Merricks a relu le texte «At the Landing" dans une perspective féministe et gothique en commentant les motifs d'enfermement et de violence inscrits dans un cadre menaçant.

Géraldine Chouard (Université de Paris-Dauphine) a ensuite rendu hommage au talent de photographe de Welty en évoquant cette période de sa vie. Elle a expliqué que cette partie de son œuvre était encore assez peu connue en France mais que le public français avait pu récemment en découvrir certains aspects grâce à l'exposition d'Annie Leibovitz qui s'est tenue à Paris de Juin à septembre 2008. S'y trouvait notamment le portrait de Eudora Wetly fait par Leibovitz en 1997, qui révèle le regard d'une photographe sur une autre photographe. Les portraits de Welty sont assez rares, le plus célèbre étant sans doute celui de Jille Krementz en 1972. Mais Leibovitz a photographié Welty à la fin de sa vie, alors qu'elle n'écrivait plus. Géraldine Chouard a commenté la posture humble de Welty, assise chez elle à Jackson, et son regard presque étonné. Elle voit dans ce portrait d'une femme discrète par une photographe très médiatique, un 
hommage qui se trouve confirmé par la présence du large portrait en noir et blanc d'Oseola McCarty, "Washerwoman and Philanthropist ", prise dans le Mississippi, là où Welty avait fait l'essentiel de son reportage pour la WPA. Ce portrait rappelle beaucoup le cliché de Welty "The Woman in the Buttoned Sweater ", où une femme noire se tient devant l'objectif, fière et courageuse. Ce qui émerge finalement du portrait de Welty, et que Leibovitz a su capturer, c'est ce mélange entre vie protégée et audace intérieure, car comme l'écrivait Welty dans son célèbre essai autobiographique, One Writer's Beginnings: "Ma carrière d'écrivain est issue d'une vie protégée. Mais protégée ne signifie pas dépourvue d'audace. Tant il est vrai que toute audace sérieuse commence de l'intérieur." (Les Débuts d'un écrivain, 191) ("I am a writer who came from a sheltered life. A sheltered life can be a daring life as well. For all serious daring starts from within. » (One Writer's Beginnings, 104).

La matinée s'est achevée avec Richard Ford qui a fait une brillante lecture d'un extrait de One Writer's Beginnings et d'un de ses propres romans.

Les trois communications de la session suivante « Reading Race » ont tourné autour de la question de race chez Welty. Dans le roman Delta Wedding, Sarah Ford (Baylor University) a souligné le pouvoir subversif de l'humour qui renverse les relations de pouvoir en dévoilant le point de vue Afro-Américain. Et David McWhirter (Texas A\&M University), comparant les personnages d'Afro-Américains à des "agents secrets », a rappelé que Welty n'a pas hésité à les représenter directement (par exemple dans " Powerhouse ») mais qu'ils résistent à l'interprétation, ou sont curieusement absents, comme dans "The Demonstators». Eudora Welty garde le secret, laissant le lecteur lever « le voile » pour chercher la vérité. C'est ce que Rebecca Mark (Tulane University) a essayé de faire en posant l'hypothèse que dans "The Demonstrators ", le pouvoir Noir remplace la violence dans le récit. Son intervention a soulevé un débat intéressant entre les partisans du caractère opaque du texte weltien qui, avec Harriet Pollack, parlent de "stratégies d'obstruction", et ceux qui, comme Rebecca Mark, maintiennent que, même si les récits sont difficiles, la clé est toujours donnée par Welty.

La dernière session de la journée "Revisiting The Golden Apples » a été naturellement consacrée à ce magnifique recueil. Carey Wall (San Diego State University) a étudié le rapport complexe de Virgie Rainey avec la communauté de Morgana. En revenant à la mort de sa mère, Virgie réaffirme le pouvoir de la vie sur la mort. Et c'est parce qu'elle regagne la communauté qu'elle peut alors en repartir et redevenir étrangère; l'épilogue n'est pas une fuite mais une transformation. Debra Dobkins (Brenau University) a ensuite justement comparé les symboles de transformation dans la nouvelle "The Wanderers » et le poème de Yeats "The Song of Wandering Angus ». Comme Carey Wall, elle a commenté le retour de Virgie dans la communauté humaine et sa métamorphose. Pour Dobkins, le récit est parsemé de seuils et d'espaces liminaux, et le bain de Virgie dans la rivière au crépuscule est hautement symbolique : Virgie, purgée de sa culpabilité, est réunie avec elle-même, avec l'amour, et l'humanité. Pourtant, elle refuse la plénitude : en devenant la fille du soleil, de la lune et des étoiles, elle se libère des attaches qui la retenaient jusque-là. Ruth Weston (University of Tulsa) a également poursuivi la notion de lien dans The Golden Apples en écoutant les voix narratives de la nouvelle «Music From Spain » et en soulignant la relation organique entre le contenu du récit et sa forme. La voix du narrateur est liée à l'esthétique du récit, et ses récits imbriqués. La scène est vue à travers les yeux d'Eugene et le récit est 
raconté par ses voix intérieures qui s'expriment, de manière paradoxale, silencieusement à travers ses pensées et ses émotions, les différentes voix articulant les motifs opposés d'enfermement et de liberté. Weston parle ainsi d' «explosion silencieuse ", ou plutôt d'implosion, pour qualifier les moments où l'expression devient impossible chez Welty.

\section{9 avril} accompagné cette Conférence du Centenaire : une représentation de The Ponder Heart au New Stage Theatre suivie d'une passionnante discussion avec le metteur en scène et les acteurs, un concert au Thalia Mara Hall avec certains des morceaux préférés de Welty et une illustration musicale de la nouvelle «The Whistle », enfin, une visite de Jackson conduite par Suzanne Marrs qui s'est achevée de façon émouvante devant sa tombe au cimetière.

Mais c'est surtout l'exposition de photographies au Musée d'Art Moderne, «Eudora Welty in New York ", reproduisant l'exposition de 1936 de ses photographies, qui fut l'événement de ces quatre jours. Car même si Welty avait abandonné le métier de photographe pour l'écriture, elle avait d'abord été reporter pour la WPA (Work Progress Administration) dans les années trente, sillonnant le Mississipi mais aussi New York. Ses clichés sont des témoignages saisissants de la Dépression et des portraits humains à la fois intimes et universels. L'exposition, organisée par Sean Corcoran, conservateur des photographies (photography curator) au Musée de la ville de New York, est donc venue à 
Jackson pour lui rendre hommage. Les photographies exposées, publiées pour la plupart dans le célèbre recueil One Time, One Place, reproduisaient celles de l'exposition originale de Madison Avenue à New York en 1936, avec à côté des photos du Mississipi, des clichés New York, pris vers 1935 au cours d'un des nombreux séjours de Welty dans la mégalopole américaine. Ces clichés témoignent eux aussi de la Dépression, mais avec un visage résolument urbain.

Les photos n'étaient pas affichées dans le même ordre qu'en 1936: elles ont été agencées de façon à suivre le projet d'un livre de photographies de Welty jamais publié, "Black Saturday », dont le but était de saisir l'essence d'une journée dans le Sud.

Et c'est en effet le rythme d'une journée d'école et de travail qui se dessine à travers les photos «Schoolchildren» et «Storekeeper». Mais plus souvent, ce sont les personnages dans leurs moments de loisirs qui intéressent Welty : les samedi passés sous le porche à se reposer ("Saturday Off, Jackson»), à faire des courses en ville ("Window shopping / Grenada»), ou encore les défilés, carnavals et fêtes foraines ( "Bird Pageant costumes / Jackson » et « Hypnotist, State Fair / Jackson »). Les gens sont très rarement photographiés chez eux, plus souvent dans la rue (par exemple "Sunday school child / Jackson») ou sous le porche de leurs maisons ("Staying Home / Jackson »), comme si la pudeur de Welty l'empêchait de franchir le seuil et de pénétrer leur intimité.

L'aspect le plus frappant de cette exposition est sans aucun doute l'impression de dialogue qui se dégage de ces photos, certaines semblant se parler, se faire écho et se répondre... Par exemple, la photographie "The Date », où l'on observe un homme et une femme qui bavardent dans la rue, est juxtaposée à « Stood Up » où une autre jeune femme attend, seule, dans une gare. Et encore "Small Talk» et "The Boast », avec ses hommes qui plaisantent et se racontent des histoires. Autant d'images fixes et silencieuses qui parlent cependant le langage des émotions, des sentiments, et peuvent être lues comme un véritable récit.

36 Une photographie se détachait de toutes les autres et interpellait le spectateur, «Mother and child / Hinds County» (qui fait partie du recueil de photographies One Time, One Place) : une femme noire y est photographiée de profil devant le porche d'une maison, la tête tournée vers l'objectif, avec son enfant sur le dos. Le petit garçon regarde l'appareil, ses yeux sont fixés, presque hypnotisés par l'objectif tandis que le regard de la mère, semble porter bien au delà, révélant à la fois la détresse et la détermination, en même temps que le lien très fort entre « mère et enfant ». Ce portrait est tout à fait caractéristique du regard que Welty elle-même portait sur ses sujets : à la fois distancé, refusant de s'immiscer dans leur intimité, et humain, rempli d'admiration pour ces gens si courageux en période d'adversité. La compassion que l'on peut ressentir devant ces photos n'est pas volontaire, à la différence de celles de Walker Evans prises à la même période, et cela tient sans doute au fait que la plupart des clichés sont pris sur le vif. On éprouve pour cette femme et ce petit garçon un fort sentiment d'empathie, mais qui n'est pas de la pitié.

Cette exposition qui ramenait l'œuvre photographique de Eudora Welty dans sa ville natale, a attiré de nombreux habitants de Jackson venus redécouvrir leur histoire. On peut d'ailleurs noter l'intérêt grandissant de la critique, ces dernières années, pour les photographies de Eudora Welty. département d'archives, le M.D.A.H (Mississippi Department of Archives) où se trouve la 
plus large collection de manuscrits, lettres, et photographies de Welty. Le chercheur comme le lecteur passionné de Eudora Welty peut y consulter une importante quantité de documents rares et d'un intérêt considérable. La salle Media regroupe tous les documents audio (enregistrements de lectures etc.) tandis que les documents visuels, y compris les photographies, sont accessibles dans la salle des Archives (Archival Reading Room). Pour y accéder, il suffit d'en faire la requête sur le catalogue en ligne des Archives, puis de venir récupérer les pièces demandées dans la salle même. Enfin, signalons qu'on peut faire des recherches à l'avance via le site Internet, et y trouver des documents uniques.

\section{Departement des Archives (MDAH)}

\section{BIBLIOGRAPHIE}

MARRS, Suzanne, Eudora Welty : A Biography. New York : Houghton Mifflin Harcourt, 2005.

WELTY, Eudora, One Time, One Place. Mississippi in the Depression. A Snapshot Album. New York: Random House, 1971.

-------, One Writer's Beginnings. Cambridge : Harvard University Press, 1984.

-------, Les Débuts d'un écrivain. Paris : Flammarion, 1989. (Traduction Michel Gresset) 
INDEX

Thèmes : Trans'Arts

AUTEUR

ISABELLE MATTÉI

Université de Versailles St Quentin, doctorante 